\title{
Jumping to a Conclusion: Fallacies and Standards of Proof
}

\section{Douglas Walton}

Centre for Research in Reasoning, Argumentation \& Rhetoric

University of Windsor

c/o Canterbury College

2500 University Ave. W.

Windsor, Ontario

Canada N9B 3Y1

dwalton@uwindsor.ca

\section{THOMAS F. GORDON}

\section{Fraunhofer FOKUS}

Berlin, Germany

thomas.gordon@fokus.fraunhofer.de

\begin{abstract}
Five errors that fit under the category of jumping to a conclusion are identified: (1) arguing from premises that are insufficient as evidence to prove a conclusion (2) fallacious argument from ignorance, (3) arguing to a wrong conclusion, (4) using defeasible reasoning without being open to exceptions, and (5) overlooking/suppressing evidence. It is shown that jumping to a conclusion is best seen not as a fallacy itself, but as a more general category of faulty argumentation pattern underlying these errors and some related fallacies.
\end{abstract}

Résumé: Cinq erreurs tombent sous la notion de conclusion hâtive: (1) avancer des prémisses insuffisantes pour établir une conclusion, (2) employer des sophismes qui font appel à l'ignorance, (3) tirer une mauvaise conclusion, (4) employer des arguments réfutables sans être ouvert à des exceptions, (5) négliger/supprimer des preuves. On démontre qu'il est préférable d'envisager des conclusions hâtives non pas comme des sophismes, mais plutôt comme une catégorie générale d'argumentation défectueuse qui inclut ces cinq erreurs ainsi de quelques sophismes reliés.

Keywords: defeasible reasoning, classification of fallacies, ignoring exceptions, secundum quid fallacy, burden of proof, errors of reasoning, suppression of evidence

\section{Introduction}

One common explanation in logic textbooks why several of the traditional informal fallacies are held to be fallacious is that the arguer

(C) Douglas Walton and Thomas F. Gordon. Informal Logic, Vol. 29, No.2, nn $2.15-743$ 
jumps too quickly to a conclusion that is not justified by the premises of the argument. The fallacy called hasty conclusion, also called hasty generalization (Walton, 1999), ignoring qualifications, etc., ${ }^{1}$ seems to centrally fit this kind of error. As shown in this paper, four other informal fallacies fit this category as well: post hoc, ad ignorantiam, ignoratio elenchi, ${ }^{2}$ and suppressed evidence. It is shown how each of these distinct kinds of error of reasoning can be classified under the more general heading of the error of leaping to a conclusion too quickly. In this paper, four distinct types of error associated with these fallacies are identified and classified. Some general lessons of these findings for fallacy theory are also drawn, relating to problems of how to classify fallacies, how to define the concept of fallacy, and how to better treat some controversial examples. One problem is that these four informal fallacies seem to involve some form of jumping to a conclusion, but the errors occur in different ways. This raises a problem not only of classifying the various fallacies, but of seeing whether all or some of them are based on some larger underlying category of faulty reasoning associated with jumping ahead too quickly to a conclusion.

We analyze a number of standard examples of such arguments of familiar kinds taken in the logic textbooks to represent informal fallacies. Our first pass is to analyze these arguments by means of tools commonly used in logic, like argument diagramming methods, that identify the premises and conclusions of an argument, missing assumptions in an argument, and chains of argumentation in which one argument is connected with another. Our finding is that these methods do help us to identify the types of arguments represented by the examples, and to grasp some normative conditions of their use and misuse. However, despite the usefulness of these methods in posing the problem in a more specific way, we show that the project of analyzing these fallacies outruns them. We show that these fallacies need to be understood as violations of procedural norms of a reasoned discussion or investigation called a dialog.

The solution we offer is based on a formal model of proof standards and burden of proof (Gordon and Walton, 2008) built on earlier research in artificial intelligence (Gordon, Prakken and Walton, 2007) on burden of proof. In this model, a dialog is defined as a triple $\langle O, A, C\rangle$, where $O$ is the opening stage, $A$ is the argumentation stage, and $C$ is the closing stage. A burden of proof is set at the opening stage for each of the two parties in the dialog, comprising a thesis (designated proposition) that each party must prove, in order to "win" at the closing stage, and a standard of proof that must be met. The notion of having different proof standards for arguments depending on a type of dialog was inspired by

\footnotetext{
${ }^{1}$ The terminology is by no means standardized (Hamblin, 1970; Walton, 1999).

${ }^{2}$ Ignoratio elenchi can be characterized as the fallacy of arguing to a wrong conclusion, but the problem is how broadly or narrowly this kind of error should be construed (Hamblin, 1970).
} 
legal proof standards that can vary. For example the preponderance of evidence standard is used in civil law, whereas the higher 'beyond reasonable doubt' standard is required in criminal law. As shown in (Gordon and Walton, 2009), such standards can be ordered by the relative amount of proof needed to satisfy the standard, going from weaker standards to more strict ones.

\section{Informal fallacies involving jumping to a conclusion}

If we look over examples of the informal fallacies presented in logic textbooks, we see that many of them, no matter how they are classified, show the distinctive sign that they appear to be fallacious because they jump hastily to a conclusion not adequately supported by the premises. Johnson and Blair $(1977,17)$ cite numerous examples of such cases where the fallacy of "hasty conclusion" is committed because premises fail to provide sufficient support for a conclusion, but where an arguer jumps to that conclusion uncritically anyway. Such arguments are often based on generalizations that are stereotypes like "Fundamentalists are intolerant of other religions" (Carey (2000, 221). When such a generalization is applied to a specific case, we can get an inference like this one: "Fundamentalists are intolerant of other religions; Bob is a fundamentalist; therefore Bob is intolerant." The problem is that even if we recognize that the first premise is not an absolute universal generalization, the two premises still fail to provide sufficient support for accepting the conclusion uncritically. Bob may be a tolerant fundamentalist.

We are often warned about the dangers of this kind of thinking. The skeptical philosopher Arcesilaus held that nothing is more shameful than for assent and approval to run ahead of knowledge and perception. ${ }^{3}$ Here the arguer seems to jump too quickly to the conclusion, even if the precise nature of the error is unclear. The jump is so hasty and poorly thought out that the fallacy could equally well be called "leaping to a conclusion", because a leap sounds even more precarious that a jump. The argument is too hasty in this case, and can correctly be described as a fallacy, because it depends on an implicit stereotyping that oversimplifies and ignores contrary evidence. A pre-existing prejudice can easily make an arguer jump to an unwarranted conclusion.

Another fallacy that fits very well under this category is that of post hoc reasoning, often called "false cause". Arguing from a perceived correlation between two events to the conclusion that one causes the other is, in principle, a legitimate form of reasoning. Indeed, many inductive arguments to causal conclusions are based on correlations. The

\footnotetext{
${ }^{3}$ Cicero, Academica I-45. The rashness of assent Cicero describes as held to be something shameful by Arcesilaus equates nicely with the fallacy of jumping to a conclusion.
} 
fallacy of post hoc is said to arise when the arguer jumps too quickly to the causal conclusion, while overlooking other evidence that ought to be taken into account, and that would indicate that reservations need to be considered. The following example is categorized under the heading of the fallacy of false cause (Hurley, 2003, p. 135).

There are more laws on the books today than ever before, and more crimes are being committed than ever before. Therefore, to reduce crime we must eliminate the laws.

This argument fits one of the three species of the post hoc fallacy classified by Pinto $(1995,306)$. An analysis of the structure of the reasoning in this species of the fallacy can be built by considering the argumentation scheme for arguing from correlation to causation, along with its matching set of critical questions.

The argument scheme and a set of three of the critical questions matching it (Walton, 2006 p. 101-103) are presented below.

\title{
Arguments scheme for argument from correlation to cause
}

\author{
PREMISE There is a positive correlation between $A$ \\ and $B$.
}

CONCLUSION Therefore $A$ causes $B$.

\section{Three critical questions matching the scheme}

$\mathrm{CQ}_{1}$ : Is there really a correlation between $A$ and $B$ ?

$\mathrm{CQ}_{2}$ : Is there any reason to think that the correlation is any more than a coincidence?

$\mathrm{CQ}_{3}$ : Could there be some third factor $C$, that is causing both $A$ and $B$ ?

These three critical questions are merely presented as a simplified example of how the scheme works. A fuller list of seven critical questions is presented in (Walton, 1995, p. 142). This argumentation scheme represents the structure of a kind of argument that can be reasonable. Suppose the correlation is shown to exist, and therefore the premise of the causal argument is shown to be true. At very least, such a finding legitimately suggests that the possibility that a causal connection may exist, and could be further investigated. As noted by Pinto (1995, 306), "many authors have pointed out that the inference from correlation to cause may lend significant support to a causal hypothesis". However one of the three species of post hoc fallacy identified by Pinto is the error of jumping to a causal conclusion only on the basis of a correlation without considering other questions.

On our analysis, the first premise makes the claim that there is a positive correlation between increasing numbers of crimes being 
committed and increasing numbers of laws on the books. The interim (implicit) conclusion suggested to follow from this alleged fact is the claim that the latter increase is causing the former one. From this interim conclusion, the stated conclusion, 'To reduce crime we must eliminate the laws' is held to follow. Along with the interim conclusion there is an additional implicit premise, where $X$ and $Y$ are variables for types of events: 'If $X$ causes $Y$, then to eliminate $Y$ we must eliminate $X$ '. This generalization does not hold up, since a type of event can have multiple causes, and to eliminate something that causes the event will not necessarily eliminate the occurrence of the event (although it might). This argument can be criticized on a number of grounds, but the interesting core of it is a post hoc argument.

The following example of the argumentum ad ignorantiam is the classic foreign spy case (Walton, 1989, p. 45), where X stands for the name of a person.

Mr. X has never been found guilty of breaches of security, or of any connection with agents of the foreign country he is supposedly spying for, even though the Security Service has checked his record. Therefore, Mr. X is not a foreign spy.

It is impossible to be absolutely certain that Mr. X is not a foreign spy. He could be a "mole", like Kim Philby, the British intelligence agent who concealed his activities as a spy for the Soviet secret service for most of his working career (Walton, 1996, p. 165). Perhaps for this reason, the argument from ignorance has traditionally been classified as a fallacy in logic. After all, arguing merely from a lack of evidence doesn't really prove anything at all.

But is argument from ignorance, or argument from lack of evidence ${ }^{4}$, as it might better be called, really a fallacy in all instances? Suppose that a thorough search by a competent government security agency turned up no evidence of Mr. X's being a foreign spy. The negative evidence provided by such a search could be part of a reasonable defeasible argument for the conclusion that $\mathrm{Mr}$. $\mathrm{X}$ is not a foreign spy. Once an implicit premise has been revealed, the argument can be properly evaluated, depending on whether it is justified. A key part of the argumentation is the following depth-of-search premise: if Mr. X really was a foreign spy, the search by the competent security agency would probably have discovered some evidence of his being a foreign spy. Inserting the implicit depth-of -search premise in the foreign spy case (represented as a conditional statement in the analysis of arguments from ignorance in (Walton, 1996, p. 259)), yields the following reconstruction of the argument.

${ }^{4}$ During discussion at the ICAIL 2009 conference in Barcelona on June 14, Trevor Bench-Capon suggested a highly suitable term for this type of argument: absence of reasons to the contrary. 
If $\mathrm{Mr} . \mathrm{X}$ is a foreign spy, the search by the security agency would have discovered some evidence of his being a foreign spy.

The search by the Security Service found no evidence of Mr. X's being a foreign spy.

Therefore, Mr. X is (probably or plausibly) not a foreign spy.

This argument is defeasible, as shown by the Philby case. Even if the premises are true, it does not follow necessarily that the conclusion is true. But still, it seems to be a reasonable argument from ignorance. Fallaciousness, in such a case, depends on defeasibility, which in turn depends on an implicit premise.

A problem posed for fallacy theory stems from the increased recognition in recent times of the legitimacy of defeasible reasoning (Prakken and Sartor, 1997). This kind of reasoning does make a tentative jump to a conclusion, typically on the balance of considerations under conditions of uncertainty and lack of knowledge, of a kind that is subject to defeat (Pollock, 1995; Walton and Reed, 2002). Should new evidence come in, the conclusion may have to be retracted (Prakken and Sartor, 2003). Much recent work in AI in particular has been done on defeasible reasoning, resulting in many formal models of nonmonotonic ${ }^{5}$ reasoning, in which the adding of new premises to an argument may fail to preserve the conclusion (Horty, 2001, p. 336). Such an argument can rightly carry weight, or be a plausible basis for acceptance, on a balance of considerations in an investigation or discussion that is moving forward, as new evidence is being collected, even if it might fail as new premises are added (Rescher, 1976). Yet jumping ahead too quickly to a conclusion, before all the evidence is in, can be an error.

Defeasible reasoning of the kind that guides so much of our intelligent behavior needs to be defined, at least in part, as based on reasonable argument from ignorance. Horty (2001, p. 337) defined default reasoning as "reasoning that relies on absence of information as well as its presence, often mediated by rules of the general form: given $P$, conclude $Q$ unless there is information to the contrary". A leading theory of defeasible reasoning (Reiter, 1980) is based on the possibility of invoking what is called the "closed world" assumption, a closure rule that allows us to assume that all relevant positive information has now been collected in a case. Using this rule, it is legitimate to conclude that a positive proposition is false whenever it is not explicitly present in a database (Horty, 2001, p. 241). Such an argument is quite clearly a reasonable form of argument from ignorance used in defeasible reasoning.

\footnotetext{
${ }^{5}$ Monotonicity of an argument is defined (Horty, 2001, p. 336) as the property that if a conclusion follows from a set of premises, it will still follow if other premises are added to the original set.
} 
To illustrate the point, the following example (Reiter, 1980, 85) can be used to show how the closed world assumption is used as the basis for drawing an inference based on absence of reasons to the contrary (argument from ignorance).

A passenger in an air terminal is scanning the televised flight monitor, to see whether there is a flight from Vancouver to New York. She scans over all the flight connections listed on the monitor, and finds no Vancouver/New York flight among the flights listed. She concludes there is no flight between Vancouver and New York.

According to the closed world assumption, any positive fact not specified in a given database (the knowledge possessed by the agent) may be assumed to be false. However, it is assumed that all the relevant information in a situation has been specified, and anything else may be disregarded, or taken not to apply to the situation as known. The closed world assumption, in this example, is that all the flights one can take from this terminal at this time are listed on the monitor. The inference may then be drawn that if a flight from Vancouver to New York is not listed, there is no such flight available. Such an inference fits the form of the argument from ignorance, for if a proposition is not stated, that lack of knowledge justifies the inference that the proposition is (or may be assumed to be) false.

What is the difference between a reasonable argument from ignorance and a fallacious one? One answer (Walton, 1996) is that in the fallacious instance of the lack of evidence argument, the arguer leaps ahead too quickly to the conclusion, failing to satisfy the requirements of a depthof-search of premise, or perhaps even ignoring it entirely. One might cite the classic case of argument from ignorance used to illustrate the fallacy in the logic textbooks. The case in point is the McCarthy witch hunt investigation in which an innocent person was accused of being a communist on the grounds that there was no evidence in the file that he was not a communist. ${ }^{6}$ The problem with this kind of case may not be just its logical form as an argument from ignorance. On least one analysis, the form of the argument could be reconstructed as follows.

Major premise: If there is no evidence that not $A$ is the case then conclude $A$.

${ }^{6}$ In the early 1950s, Joseph R. McCarthy, a U.S. senator, accused many innocent people of being Communist sympathizers, with the result that they were perceived as "loyalty risks" and lost their jobs. McCarthy used the form of reasoning called argument from ignorance when he used the following pattern of argument: "There is nothing in the files to disprove this person's Communist connections, therefore we can infer that he has Communist connections." 
Minor premise: There is no evidence that not $A$ is the case.

Conclusion: $A$

This form of argument is deductively valid (modus ponens). The real problem with the witch hunt example is that the major premise of the argument reverses the burden of proof. The truth or acceptability of the major premise seems questionable, since it would require a database that keeps track of evidence for what is not the case. Hence the deeper problem is not just in the logical form of the argument, but in how burden of proof is assigned during an investigation in which data is being collected.

So far, then, we have examined three informal fallacies, hasty generalization, post hoc and argument from ignorance, that can all be analyzed as fallacies by showing that the argument jumped too quickly to the wrong conclusion. Next, it needs to be asked whether this error of having jumped to the wrong conclusion is a special case of a broader error, that of arriving at a conclusion other than the one which is supposed to be proved.

On Aristotle's theory, a fallacy is a sophistical or apparent refutation in a chain of valid syllogisms that appears to refute the conclusion it is supposed to refute, but does not. According to Hamblin's summary of Aristotle's theory (Hamblin, 1970, p. 105), such a failure can occur for any one of the following nine reasons: (i) the reality is not contradicted, but only the name, or (ii) the proof contains only a 'synonymous' word, or

(iii) the premises of the refutation are not granted, or (iv) are not necessary (but only accidental), or (v) the original point to be proved is among the premises, or (vi) the refutation does not refute in the same respect or (vii) relation or (viii) manner or (ix) time.

Each one of these failures could be classified as a separate fallacy in its own right, but violating any of requirements (vi) through (ix) amounts to committing the secundum quid fallacy of overlooking exceptions to a generalization (Walton, 2004, p. 33). It is easy to see why the fallacy of ignoratio elenchi (ignorance of refutation) came to be a catch-all category in the logic textbooks through the ages.

However, in another place Aristotle gave a much more specific definition of the fallacy of misconception of refutation, shown below in a literal translation of the following passage in Topica (162a13 162a16).

When the argument stated is a demonstration [apodeixis] of something, if it's something other than that leading to the conclusion, it will not be a syllogism about that thing. ${ }^{7}$

\footnotetext{
${ }^{7}$ Translation provided by Craig Cooper, December, 1995), as quoted in (Walton, 2004, p. 35).
} 
The fallacy described here could fit the error of leaping to the wrong conclusion quite well. The problem is that the given argument may prove a conclusion. Thus it might appear to be a good proof, and it might even be valid. But it is a fallacious argument if it did not prove the conclusion that was supposed to be proved. This kind of fallacy is classified in (Walton, 2004) as a failure of relevance. An argument may be valid, but if it goes to a conclusion other than the one that is supposed to be proved, it is irrelevant. The problem is how widely or narrowly relevance should be defined.

The following example is classified under the heading of the fallacy of ignoratio elenchi translated as "missing the point" (Hurley, 2003, p. 123).

Crimes of theft and robbery have been increasing at an alarming rate lately. The conclusion is obvious: we must reinstate the death penalty immediately.

The clue to the reader in this case that enables him or her to see that the argument in the given case is a fallacy is the hasty jump to a conclusion that seems almost ridiculously inappropriate. It is the wrong conclusion. But should the diagnosis be that the argument is irrelevant, or simply that it is too weak to adequately support its conclusion? Maybe neither is the heart of the problem. A better analysis might be that the argument ignores or suppresses evidence that is relevant, and that should be taken into account. Reinstating the death penalty is a solution that arguably won't work to stop crimes of theft and robbery, at least so many would say. At best it is an extreme solution, and other possible solutions should also be considered before leaping to this conclusion. As in the example of post hoc cited above, the argument seems fallacious because it overlooks or suppresses evidence on crime prevention that ought to be taken into account.

Another error in this case is that theft and robbery would not be punishable by death, even if the death penalty were reinstated. Thus reinstatement cannot be expected to deter theft or robbery. On this analysis, one might question whether the argument is an example of jumping to the wrong conclusion, so much as it is an example of including some implicit assumptions that are questionable and even implausible. Still, when you look at the example as stated, what stands out is the wild leap from a premise that might be true to a conclusion that doesn't follow.

The majority of logic textbooks don't recognize a special type of fallacy in which evidence is overlooked or ignored, making an argument too weak to support its conclusion. However, a widely used textbook (Hurley 2003) does recognize a fallacy of this kind, called the fallacy of suppressed evidence (pp. 153-155), possibly picking it up from the fallacy called "suppressed evidence" in (Kahane, 1971, 4-7). Hurley classifies this fallacy as a failure to meet a criterion of what he calls a 
cogent argument. He defines a cogent argument as an inductive argument with good reasoning and true premises (p. 153). On his analysis, quoted below, the fallacy of suppressed evidence is committed by an argument that fails to meet this requirement.

The requirement of true premises includes the proviso that the premises not ignore some important piece of evidence that outweighs the presented evidence and entails a very different conclusion. If an inductive argument does indeed ignore such evidence, then the argument commits the fallacy of suppressed evidence.

Hurley offers the following example (p. 153) to illustrate this fallacy. Let's call it the Little Dog argument.

\section{The Little Dog argument}

Most dogs are friendly and pose no threat to people who pet them. Therefore, it would be safe to pet the little dog that is approaching us now.

This example is an interesting one, but there are some problems with how Hurley uses it to define the fallacy of suppressed evidence. On his account, the fallacy of suppressed evidence is committed by an argument that ignores some important piece of evidence that outweighs the presented evidence, where the addition of the ignored evidence leads the argumentation to a different conclusion. One problem with this example is that the argument is quoted from Hurley above seems reasonable, unless there is some evidence that this particular little dog might be in some way unusual or even dangerous. Perhaps the fallacy is to overlook this possibility, given that touching an unknown dog can sometimes be dangerous. The problems are whether this failure should be seen as a fallacy or not, and if it is supposed to be a fallacy, how the fallacy should be analyzed as a distinctive type of failure or deceptive tactic. It certainly is reasonable to set a general requirement in place to the effect that all relevant evidence must be considered when evaluating the acceptability of a claim. However, this requirement does not seem to be captured in the logical form of an inference in the case of an example like the little dog argument. Instead, it seems to be a general procedural requirement on how to collect and apply evidence when evaluating an argument.

These examples are good ones for a textbook on informal fallacies that is to be used in trying to teach beginning students of logic how to recognize fallacies and evaluate arguments as reasonable or fallacious. But what is of interest is what is common to them. This particular characteristic of jumping to a wrong conclusion is evident not only in the many examples of informal fallacies given in Hurley (2003), but in the examples presented in many other informal logic textbooks as well. These observations suggest that this particular characteristic of jumping 
too quickly to a conclusion may be centrally important somehow not only for identifying fallacies, but in regard to defining and explaining the basic notion of fallacy itself.

The first premise of the little dog argument is a generalization that appears to be open to exceptions, and the argument in this example is a paradigm case of defeasible reasoning. As noted above in this section, overlooking exceptions was recognized by Aristotle as a subcategory of fallacies coming under the general heading of ignoratio elenchi. As noted in connection with Aristotle's general definition of this fallacy, violating any of requirements (vi) through (ix) amounts to committing the secundum quid fallacy of overlooking exceptions to a generalization.

\section{The Little Dog argument}

A recurrent problem in fallacy theory is that of drawing the line between examples in which the argument is merely weak, and presents insufficient evidence, and examples where it should properly be classified as fallacious. The difference is between an argument that is merely weak, or lacking adequate support, and one that commits a serious enough kind of recognizable error that it can properly be evaluated as fallacious (Walton, 1995, p. 260). The specific problem addressed in this paper is whether it is a fallacy to leap ahead too quickly to a conclusion in an argument, or even worse, to ignore or suppress evidence needed to prove its conclusion. Are such errors better classified as fallacies or as weak arguments? The Little Dog argument is surely an outstanding example that poses this question in an acute form.

One problem with the Little Dog argument as it stands is that the allegedly suppressed evidence is not stated as part of the argument. For purpose of discussion and further analysis, let's modify the argument and make the error more explicit, and easier to pinpoint and diagnose. Suppose that as we approach the little dog, we see that it looks like a pit bull, and that based on common knowledge, we know that pit bulls are dangerous. We could call this revised version of the example the Little Dog argument, version 2 or the Pit Bull argument. It does strongly seem to be fallacious. One premise of version 2 states that most dogs are friendly and pose no threat to people who pet them. But the top premise identifies the dog as looking like a pit bull. The fallacy of sticking with the previous conclusion to go ahead and pet the little dog resides in the failure of the new version of argument to take into account the new data. We see that the little dog looks like a pit bull. Based on argument from appearance, it would be fair to conclude that it might be a pit bull, for all we know. Ignoring this perceptual evidence would lead to the wrong conclusion that would be safe to pet the little dog approaching us now. Version 2 makes the example much more convincing as an argument that can properly be said to be fallacious and that ignores or suppresses evidence. 
However the question remains whether it is appropriate to call this kind of failure a fallacy. Is it more justifiable to classify it as merely a weak argument that leads to a wrong conclusion? There is no consensus in the logic text books to guide us, except that the majority of logic textbooks do not include the fallacy of suppressed evidence under the common lists of informal fallacies. ${ }^{8}$ As noted above, the issue depends on the prior unresolved issue of how to define the notion of fallacy.

Version 2 of the Little Dog argument represents a departure from version 1 by adding new evidence to the case, namely the observation that the little dog looks like a pit bull. But as indicated in the analysis represented in Figure 2 below, part of the original example was an assumption that we don't know whether the little dog is friendly or not. It was this lack-of-knowledge premise that made the argument defeasible and that also made it fall into the category of an argument from ignorance. To bring out these factors, and at the same time illustrate a different approach to argument analysis and diagramming, we diagram another version (version 3) of the Little Dog argument using the Beardsley-Freeman system method of argument diagramming supported by the Araucaria software (Reed and Rowe, 2004). This system has a number of useful features. It can used to display the difference between linked and convergent arguments, it can be used to represent implicit premises in an argument, and it can be used to represent argumentations schemes of various kinds on the diagram.

We will carry out the analysis in two parts. First we present an analysis of the original argument, which concludes that it is safe to pet the little dog. Then we present the secondary argument that attacks the original argument. We begin the analysis by setting out a list of the propositions (key list) in the original argument of version 3 of the little dog argument.

\section{Key list for the original argument in the Little Dog example}

- Most dogs are friendly and pose no threat to people who pet them.

- Here is a dog approaching us now.

- This dog is friendly and poses no threat to people who pet it.

- If a dog is friendly and poses no threat to people who pet it, it is safe to pet it.

- It is safe to pet this dog approaching us now.

- Let's go ahead and pet this dog approaching us now.

The diagram in Figure 1 shows the original argument used to justify the conclusion to go ahead and pet the little dog.

\footnotetext{
${ }^{8}$ It was not recognized in the account of the standard treatment of fallacies presented by Hamblin (1970).
} 


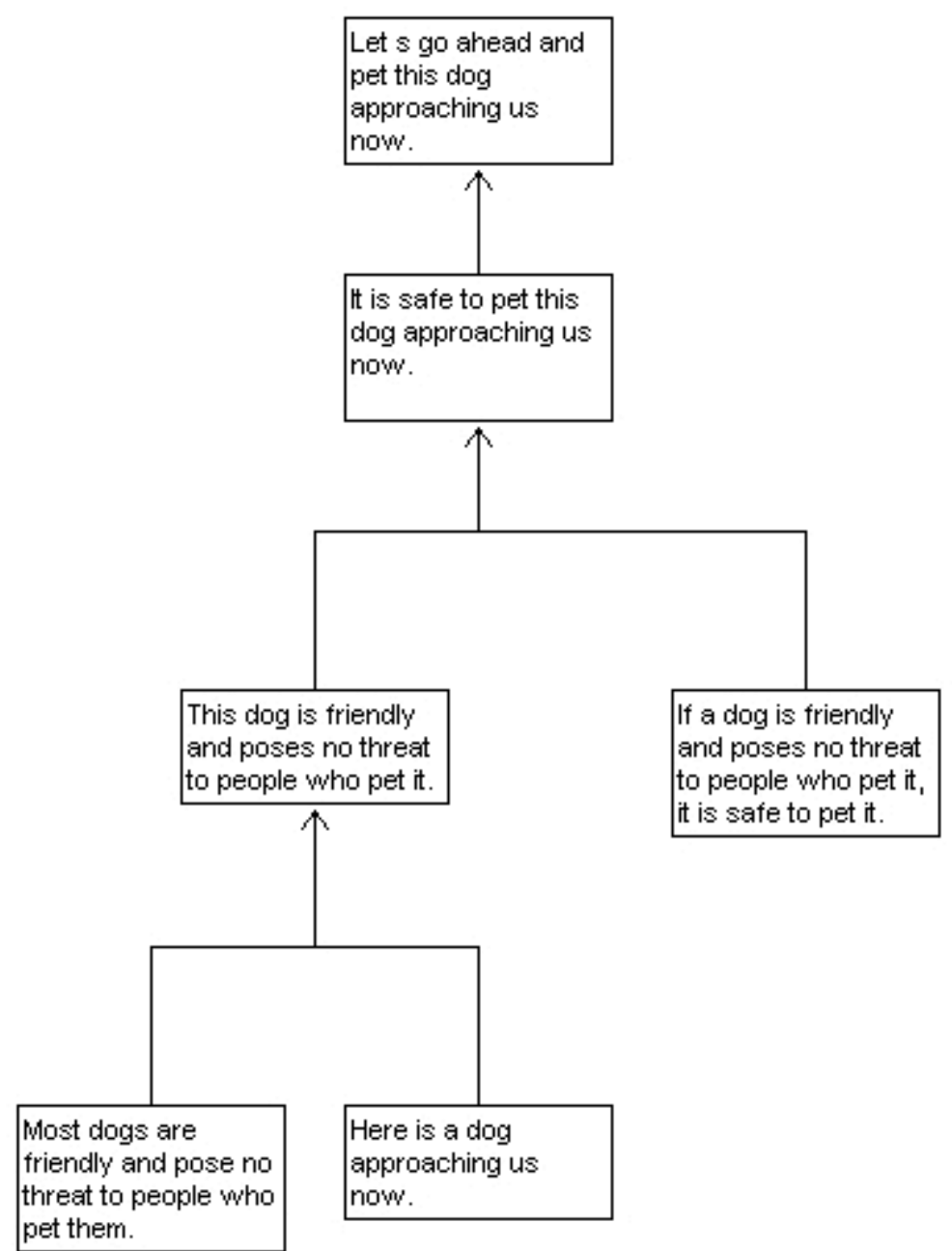

Figure 1: Araucaria diagram of the original argument in version 3

The leftmost box at the bottom of Figure 1 contains a generalization. It could be stated as an implicit premise that there is no evidence known so far that indicates that the dog approaching us now is not friendly and poses a threat to people who pet it. Inserting such a premise would make the argument an argument from ignorance. The argumentation scheme for that type of argument could be displayed on the diagram, linking the implicit premise to other premises in the diagram at the appropriate places. But rather than analyzing the argument this way, we choose an alternative method.

In the key list below, a general principle is enunciated that is taken to be an essential assumption in version 3 of the secondary argument. The principle of tutiorism comes from the doctrine of probabilism in Catholic moral theology, a part of casuistry, a method for deciding what to do when applying general ethical principles to particular cases (Jonsen and Toulmin, 1988). The rule of probabilism holds that when there is a 
preponderance of evidence on one side of a controversy, one should act in accord with the contention of that side. ${ }^{9}$ The doctrine of tutiorism is an exception to the rule of probabilism to be applied in the case where danger and the risk of error are involved. The rule of tutiorism holds that in a case of doubt one should act in accord with the contention of the safer side.

Casuistry has been discredited as a method for ethical decisionmaking, but Jonsen and Toulmin (1988) maintain that it had a lot of very useful techniques that were unfairly attacked. The casuistic language of probabilism is, however, misleading in some respects. When casuists say that something is probable, they do not refer to probability in the modern sense, and it might be better to use the term 'plausible' to express this key notion. Reformulated in these terms, the two rules could be less misleadingly formulated as follows. The first is a general rule that one should act in accord with the view that is more plausible. There is a secondary rule that applies in a case in which safety is at issue. The secondary rule allows that in such cases it may be reasonable to act in accord with a less plausible view. It is this secondary rule that best formulates the principle of tutiorism.

\section{Key list for the secondary argument in the Little Dog example}

- Exception: some dogs are not friendly and pose a threat to people who pet them.

- If a dog is not friendly and poses a threat to people who pet it, it is not safe to pet it.

- It is not known whether this dog approaching us now is friendly, and poses no threat, or is not friendly, and poses a threat.

- It may not be safe to pet this dog.

- Principle of Tutiorism: Under conditions of uncertainty and lack of knowledge, if there is a choice between one of two hypotheses, it may be reasonable to accept the less plausible one if (a) accepting the more plausible one may have significant negative consequences that are known, while (b) accepting the less plausible one does not have significant negative consequences that are known.

- Let's refrain from petting this dog.

Using this key list, the secondary argument in version 3 is analyzed and represented in Araucaria as shown in the argument diagram in Figure 2.

\footnotetext{
${ }^{9}$ Other accounts of probabilism in casuistry offer differing versions of the rule - see for example the account given in the Wikipedia entry for 'probabilism', and also the discussions in (Jonsen and Toulmin, 1988). However, this is the version we find most useful here.
} 


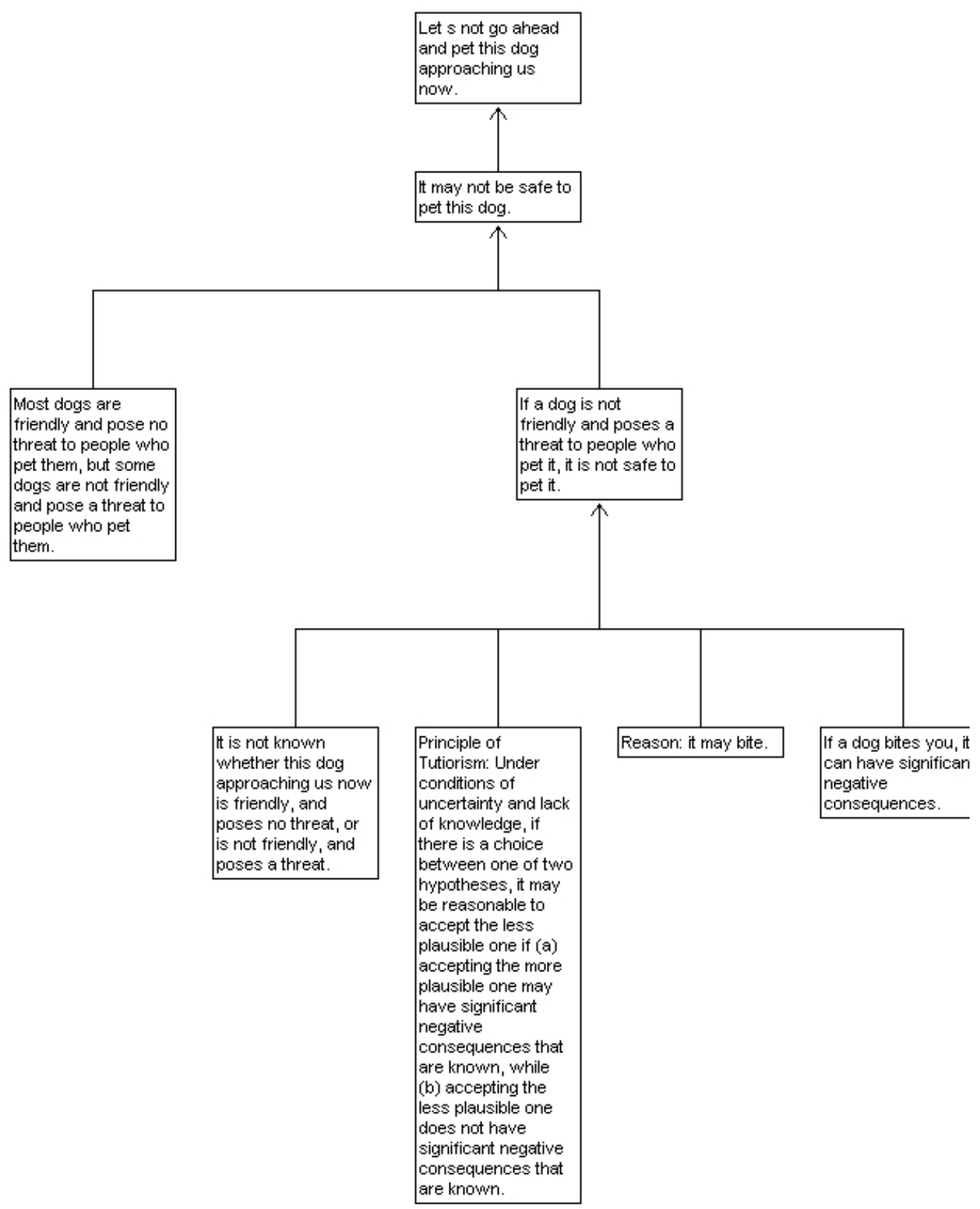

Figure 2: Secondary argument in version 3 defeating original argument

Araucaria represents refutation using a double arrow from one node to another. In Araucaria, refutation is seen as similar to classical negation in logic. In other words, it is comparable to the notion of the defeating rebutter, as opposed to an undercutting rebutter. The concept of one argument undercutting another one cannot, at least technically, be represented on an Araucaria diagram.

To represent the entire sequence of argumentation in version 3 of the Little Dog example, we need to join the two argument diagrams together 
in Figure 1 and Figure 2. We can do this by joining the ultimate conclusion at the top of the diagram in Figure 2 by means of our refutation double arrow to the ultimate conclusion at the top of the diagram in Figure 1. One is the negation of the other. The large diagram composed of the joining together of these two diagrams represents the whole sequence of argumentation in version 3, showing how the secondary argument refutes the original argument.

The main problem with this analysis is that the principle of tutiorism has been represented as a premise in the argument. This way of proceeding is okay as far as it goes, but it does not recognize that the principle of tutiorism is better seen as a meta-level procedural principle of practical reasoning that can be applied to cases of deliberation or danger, including when the danger of making an error that has significant negative consequences is involved. In such cases, a decision has to be made between accepting two equally plausible propositions, or cases where one is more plausible than the other, but where selecting the less plausible has significant consequences. Such a case brings argumentation from negative consequences into play, when a principle of risk rules against selecting the proposition representing the course of action that has the significant negative consequences. The problem with the analysis represented in Figure 4 is that although it depicts the inferential structure of the argument in a useful way by expressing the principle of tutiorism as a premise, really how this principle works in the argument needs to be seen in a different way.

It is our contention that the principle of tutiorism needs to be seen as a design principle of a class of argumentation schemes. The schemes in this class are variations and specializations of: "if $X$ is dangerous/risky then avoid $X$." An example: avoid things that look like snakes (because the cost of being bitten is higher than the cost of walking around the object). Such schemes are different from schemes based on defeasible generalizations about what is usually or normally the case, like the classic example 'Birds fly'. This does not fit the principle, since there are no apparent risks in wrongly inferring that something is or is not a bird.

In the cases we have considered, the principle of tutiorism applies under conditions of decision-making under uncertainty and lack of knowledge, where significant negative consequences of an action are involved and safety is a value. When evaluating a chain of argumentation, the principle tells us that in addition to the plausibility of the claim on each side of the controversy, practical matters of avoidance of harm need to be taken into account in decision-making. Practical reasoning concerning the goals and values of the decision-maker and a wider audience needs to be factored into the argument evaluation. A formal model that applies to cases of practical reasoning where the argumentation is based on values such as safety is that of (Atkinson and Bench-Capon, 2007). Their model applies to cases in law where decisions require an element of choice that depends on the goals and interests of the people making the decision. Their Value-Based 
Argumentation Framework (VAF) labels argument trees with the values that would be promoted if the argument were to be accepted. Thus their analysis applies to cases where safety is a value, like the Little Dog example.

\section{Four kinds of error distinguished}

There are four kinds of error that we have analyzed that need to be clearly defined, and distinguished from each other, and from other kinds of error. The first one is to argue from premises that are by themselves insufficient as evidence to prove the conclusion that is supposed to be proved in the given case. It is assumed that in the given case there is some standard of proof that indicates the party responsible and sets a standard regulating how much evidence there needs to be in order to successfully prove the conclusion. This assumption is in turn based on a prior assumption that argumentation in the given case takes place in three stages (Gordon and Walton, 2008). There needs to be an opening stage, an argumentation stage and closing stage. The global burden of proof (called the burden of persuasion in law) needs to be set at the opening stage. It is fixed throughout all three stages. This assignment of burden of proof sets the following requirements into place. (1) A thesis (a proposition) that each party has to prove is determined. (2) A standard is set determining how strong the argument of each side needs to be in order to qualify as a proof of its thesis. There is also a local burden of proof (called a burden of production in law) that can shift back and forth from one side to the other during the argumentation stage. (3) At the closing stage it needs to be decided which of the two sides met its assigned burden of proof in its argument put forward during the argumentation stage. Assuming that the thesis of one side is the opposite of the thesis of the other side, only one side can fulfill its global burden of proof.

In analyzing the four kinds of error, we have used argument diagrams to represent the inferential structure of the reasoning during the argumentation stage. However, in each of these cases we've seen that the errors are procedural. For example, in the case of the McCarthy argument, the error was a reversal of the burden of proof. In other cases as well, the error resulted from a deficiency in a search made for both positive and negative evidence, and this too was a procedural error concerning misapplication of burden of proof. It is possible to represent failure to meet a burden of proof as shown in Figure 3. 


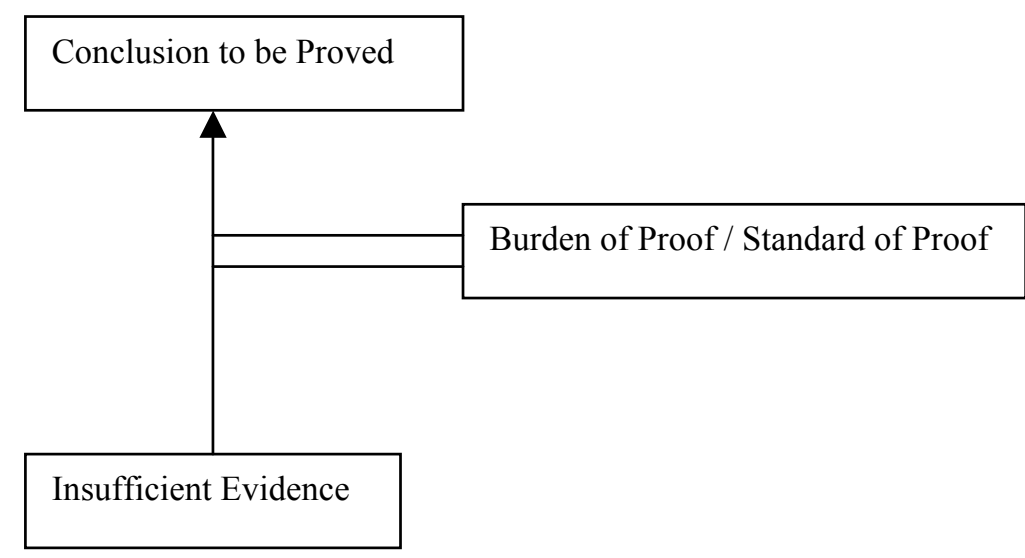

Figure 3: Failure to meet burden of proof and standard of proof requirements

In this kind of case, the arguer jumps ahead too quickly on the basis of a set of premises that is not sufficient as evidence to prove the conclusion. The problem in this kind of case is to judge whether the failure is a fallacy or simply an argument that is too weak. As this kind of problem can only be solved, we argue, by looking into procedural matters concerning the three stages of the argumentation in the context of an investigation that is supposed to arrive at the conclusion.

The second kind of error is that of argument from ignorance. As shown by the foreign spy case in Section 1, this type of argument can sometimes be reasonable, but can commit a fallacy by leaping ahead too quickly and ignoring the depth-of-search premise. The problem is that such a failure to take all the required evidence into account is a failure to meet requirements of burden of proof, making the argument too weak to prove the conclusion it is supposed to prove. Once again we could represent the error by looking at the structure of an inference from premises to a conclusion. So depicted, the general failure could be classified as a special instance of the error shown in Figure 4. 


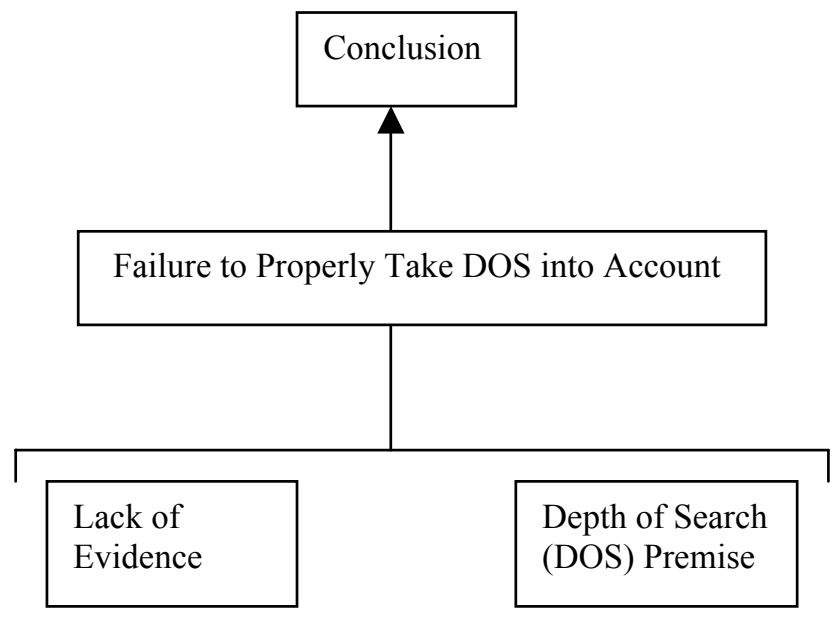

Figure 4: Fallacious argument from ignorance

The fallacy exhibited in Figure 4 fits the pattern of the error of leaping ahead too quickly to the conclusion that is supposed to be proved, because proving that conclusion needs to be based on two premises. If the DOS premise is ignored or insufficiently justified, the arguer may leap ahead too quickly and draw the conclusion merely from ignorance. But, on the other hand, it is a special kind of error in its own right, based on a lack-of-evidence premise and another premise that concerns the depth of search of the investigation used to find evidence in the case. The special kind of error is procedural, because matters of depth of search for burden of proof need to be decided at the opening stage.

The third kind of error is one where the arguer leaps to the wrong conclusion on the basis of given premises instead of constructing a line of argument that ends in the conclusion that was properly supposed to be proved in the given case. This kind of error can be shown in Figure 5.

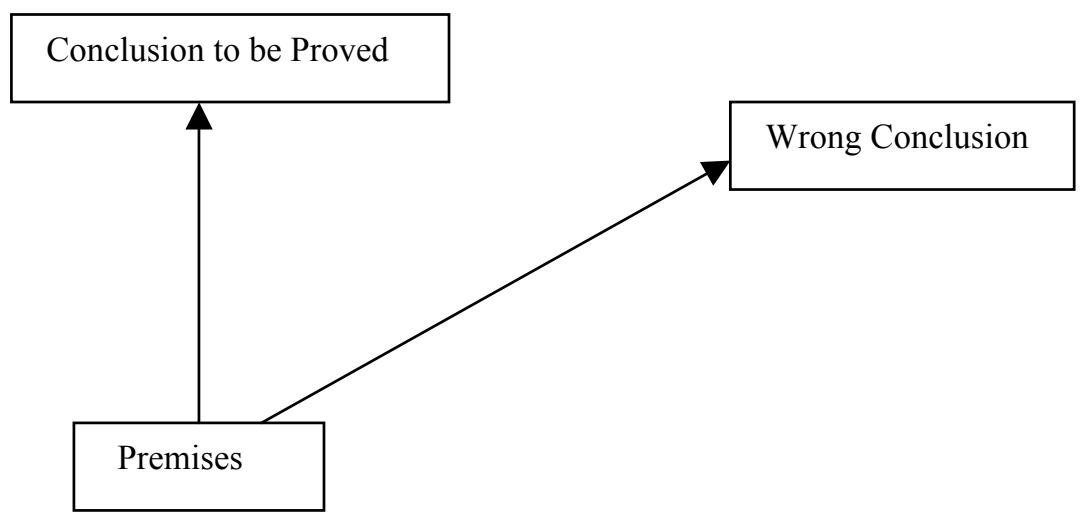

Figure 5: Leaping to the wrong conclusion 
This third kind of error, shown in Figure 5, corresponds to Aristotle's fallacy of irrelevant conclusion. This too seems to be a procedural kind of error, assuming that what determines relevance of an argument is the issue set at the opening stage of a dialog.

The fourth kind of error occurs in a case where a defeasible generalization is used along with other premises to argue for a conclusion that is supposed to be proved. In such a case, as shown in Figure 6, the inference from the premises to the conclusion is a qualified one. It is supposed to be open to defeat if new evidence comes in showing that the present case is an exception to the generalization. The proper line of inference is shown by the arrow on the left, while the erroneous line is shown by the arrow on the right.

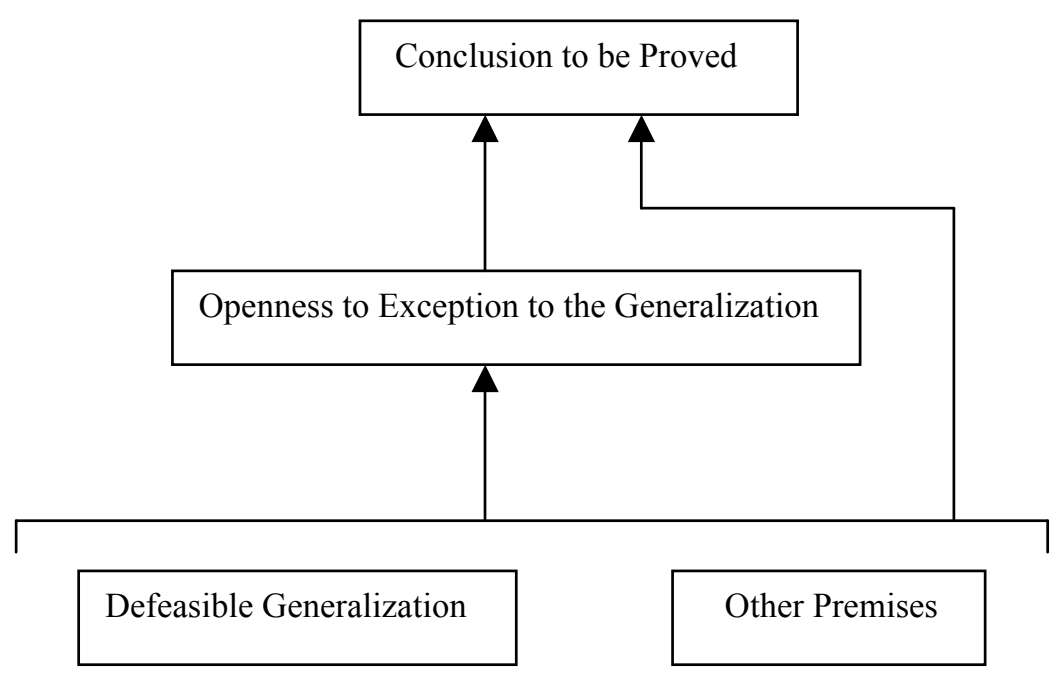

Figure 6: Jumping ahead too quickly by ignoring exceptions

In this fourth kind of fallacy the arguer exhibits a kind of closedmindedness. He or she is not open to exceptions that either do or might occur with respect to the defeasible generalization that is one of the premises. In this kind of case, the arguer leaps ahead from the premises to the conclusion, ignoring potential or actual exceptions to the generalization that need to be taken into account. From there, a decision can be arrived at on whether the error is serious enough to merit the argument being called fallacious. This kind of fallacy is often labeled under the heading of hasty generalization.

These four types of error are fundamentally important to recognize, in order to build up a fallacy theory that can classify basic errors of reasoning underlying informal fallacies that often combine the, or combine other aspects of, argumentation with an underlying pattern of erroneous reasoning. 


\section{Classifying the four types of error}

Studying these fallacies as instances of the broader error of jumping too hastily to a conclusion has raised the problem of fallacy inflation observed by Hamblin in his commentary on Aristotle's analysis of the fallacy of misconception of refutation. It is very easy to analyze not only these three fallacies, but many others as well, perhaps even including nearly all the traditional informal fallacies, as committing the fallacy of misconception of refutation. It depends on how broadly this fallacy is defined. If we define the error of arguing to our wrong conclusion broadly enough, nearly every informal fallacy could be absorbed into this model. The same remark could be said about the general category of jumping too hastily to a conclusion. The danger is that not only hasty generalization, post hoc, and argument from ignorance are fitted under this classification, but many other informal fallacies as well. Thus it would seem that the error of jumping too quickly to a conclusion represents quite a broad and general type of error of reasoning, and precise classifications of which fallacies fall under this type of error is a job that remains to be done. As Hamblin (1970) noted, attempts so far to produce a system of classification of informal fallacies have not been successful. Still, seeing how four distinct types of error are involved can help us begin this task. The analysis of the Little Dog case brought out how the study of the supposed fallacy of suppressed evidence is closely related to, and in some cases inseparable from, the fallacy of argument from ignorance. To begin the task of classifying the different ways the fallacy of jumping to a conclusion can be committed, we review and further analyze the four basic kinds of errors that could be classified under the general heading of jumping too hastily to a conclusion were distinguished in Section 4.

These four errors, it is proposed here, should be seen as specific categories of errors, corresponding to types of fallacies that have already been traditionally identified, all falling under a more fundamental pattern of faulty reasoning that that can be called jumping to a conclusion. This pattern of reasoning underlies many of the informal fallacies, and is especially closely related to the four fallacies cited in Section 1 . The first error was failure to meet a burden of proof. The problem is not only to define the notion of burden of proof, but to set standards against which a given argument can be evaluated to see whether it has met that standard of proof or not. This problem is complicated by the fact that there can be different standards for burden of proof in different dialogs, and an argument judged to be acceptable by one standard may not be acceptable judged by another. Another complication, as shown by the Little Dog example and the snake example, is that a standard of proof may be set high if the factor of safety is involved, for instance, if there is known threat to human life or the possibility of injury. The variability of standards of proof in different dialog settings has already been taken into account in law, where the burden of proof is different in a criminal trial 


\section{Douglas Walton and Thomas F. Gordon}

from a civil trial. The problem for defining failure to meet a burden of proof as a species of jumping to a conclusion is one of recognizing the variability of burden of proof in different types of dialog by setting standards appropriate for what constitutes a successful proof in a given case. Some research in artificial intelligence and law (Freeman and Farley, 1996) has drawn important distinctions between different kinds of proof standards used to set burden of proof in law.

Gordon, Prakken and Walton (2007) defined three proof standards for a statement at issue in a dialog. The idea is that after arguments pro and con have been collected together during the prior sequence of argumentation in the dialog, a decision can be taken on whether the statement should either be accepted or rejected. During the point where this decision is made in a dialog, one of the three following proof standards can be used to set in place an appropriate burden of proof against which the acceptability of the statement can be decided. A statement meets the standard of scintilla of evidence if and only if it is supported by at least one defensible pro argument. A statement meets the best evidence standard if and only if its strongest defensible pro argument outweighs its strongest defensible con argument. ${ }^{10}$ A statement meets the standard of dialectical validity if and only if it is supported by at least one defensible pro argument and none of its con arguments are defensible. The standard of beyond a reasonable doubt, one familiar from law, is another standard that might be considered as well. ${ }^{11}$

In light of the variability of such proof standards, what needs to be clarified in relation to the error of jumping too quickly to a conclusion by failing to meet a burden of proof, as represented in Figure 3, is that the failure to meet the burden of proof by presenting an argument that is insufficient to prove the conclusion that is supposed to be proved is not necessarily fallacious. The failure could be just that of an argument that is too weak to meet the appropriate standard of burden of proof, and that needs further substantiation by the bringing in of additional evidence. There is nothing inherently fallacious about such a shortcoming. Indeed, throughout this paper, we have stressed that defeasible arguments, although they often tend to be weak, should not be seen as inherently fallacious. On the other hand, certain informal fallacies are closely associated with failure to meet a burden of proof. One is the fallacy of arguing in a circle, or begging the question (petitio principii). When this fallacy is committed, an arguer fails to meet the burden of proof, when arguing for a conclusion that is doubtful, by assuming one of the premises of his argument required to be proved in order to prove that

${ }^{10}$ The preponderance of evidence standard was renamed the best evidence standard in response to a criticism (made by Trevor Bench-Capon) mentioned in (Gordon, Prakken and Walton, 2007) to the effect that the formalization of this standard proposed in that paper was not legally adequate.

${ }^{11}$ The standard of beyond a reasonable doubt was not included in (Gordon, Prakken and Walton, 2007). 
premise (Walton, 1995). But the very purpose of putting forward an argument designed to rationally persuade the questioner to accept the conclusion is to use premises that can remove the questioner's doubts about that conclusion. Committing this kind of fallacy means that the argument so used is doomed to failure, because the premise used is just as doubtful as the conclusion to be proved. Such an argument cannot effectively be used to remove the questioner's doubts about that conclusion. Although this fallacy involves a failure to meet an appropriate standard of proof, and thus is basically a fallacy of burden of proof, it is also much more than that. It is use of a circular argument of a kind that is not useful to remove doubt about a conclusion that is an issue in a dialog.

The second kind of error was closely related to the failure to meet a burden of proof by jumping ahead too quickly is the fallacy of argument from ignorance. This too is basically a failure to meet requirements of burden of proof appropriate for a dialog. But once again, the fallacy is not just the use of a weak argument that fails to meet the appropriate requirement set for burden of proof. The fallacy is one of jumping ahead too quickly, purely on a basis of ignorance, or lack of evidence, without backing one's argument up with enough additional positive evidence of the kind required to prove the conclusion. In the classic foreign spy case, the failure is that of not collecting enough evidence of the kind required to prove the depth-of-search premise. This fallacy is the error represented by the structure in Figure 4. The second kind of error of jumping ahead too quickly to a conclusion with the consequent failure to meet a burden of proof is that of the fallacious argument from ignorance, pictured in Figure 4. This fallacy, like the fallacy of begging the question, can be classified under the more general error of failure to meet a burden of proof, represented in Figure 3. However, what needs to be emphasized is that the error of failing to meet a burden of proof, as pictured by Figure 3, is not only explainable as a fallacy. It needs also to be viewed as a failure to comply with procedural norms of the type of dialogue or investigation that is underway. The fallacy is not purely in the inferential form of reasoning. The problem is that the search for arguments was not deep enough. It is like the case of a criminal trial in which the judge declares the trial to be over after the prosecution has presented its evidence but before the defense has had an opportunity to present its evidence. Or it is like the case of a deliberation about where to build a new factory in which the parties have agreed that the phase of collecting evidence will last for one week, and the moderator prematurely cuts off this information collecting phase. This kind of mistake is a procedural error, because not enough time for collecting arguments was made available.

The third kind of error coming under the heading of jumping to a wrong conclusion, represented in Figure 3, is different from errors of types 1 and 2, because it involves moving to a specific statement that is a wrong conclusion, as contrasted with the conclusion that is properly supposed to be proved in a dialog. The fallacy in this kind of case arises 
because the conclusion actually proved may appear to a respondent or an audience to be very similar to, or even equivalent to the conclusion that is supposed to be proved. This type of error, corresponding to Aristotle's fallacy of arguing to the wrong conclusion, depends on the assumption that, in a given case, a specific conclusion has been identified at the confrontation stage of a dialog as the proposition that is supposed to be proved by the arguer. Many good examples of this kind of fallacy have been cited in the logic textbooks, but we make no further comment about them here, as this type of error has already been classified as a fallacy of relevance (Walton, 1999).

It may be questioned at this point whether this third type of error properly fits under the category of jumping to some conclusion. But it does fit in, we contend, because the error is that of moving to a conclusion other than the one that is supposed to be proved. Such a movement may be a gradual or sudden, and hence it may not always the classifiable as a jump or (even less likely) a leap, and this might seem to leave open the question of whether the fallacy represented by this kind of case, a failure of relevance, should properly fall under the heading of jumping to a conclusion. Our inclination is to say that it should fit under this heading, based on the analysis of relevance in (Walton, 1999).

The fourth kind of error, and the one studied most extensively in this paper, is the error of leaping ahead too quickly by ignoring exceptions. The structure of this error was represented in the argument diagram in Figure 6. As shown there, the error is that the arguer jumps ahead too quickly from the given premises to the conclusion, ignoring the possibility of exceptions to the generalization that is one of the premises of his argument. There are two variants of this kind of error, represented by versions 1 and 2 of the Little Dog example. One is the error of ignoring or suppressing actual evidence which has already been furnished by a premise in the argument, or made available in a way that the arguer should perceive. If the little dog looks like a pit bull, and the arguer knows that a pit bull is a dangerous type of dog, overlooking the obvious appearance of the dog as being classified into the pit bull category is a serious error. This kind of error certainly could be called a fallacy, because the basis of the error is a failure of reasoning by jumping too quickly to a conclusion.

Another variant on fallacies of defeasibility that might be classified under the heading of jumping to a conclusion is the error of being closedminded by not being open to exceptions to a generalization that is part of one's argument. In Section 4 it was suggested that the arguer exhibits a kind of closed-mindedness by not being open to the possibility of exceptions, even in advance of where the particular exception has been cited by the respondent in a dialog, or is clearly visible to the arguer. One example that could fit this type of error are arguments of the sort cited in Section 1, based on stereotypical generalizations like "Fundamentalists are intolerant of other religions" (Carey $(2000,221)$. As noted in Section 1 , they can be part of an inference of this type: "Fundamentalists are 
intolerant of other religions; Bob is a fundamentalist; therefore Bob is intolerant." The problem is that if arguer's generalization is put forward in a dogmatic way, indicating that it is meant not be open even to the possibility of exceptions, it is one sort of jumping to a conclusion. The fallacy in this kind of case could be diagnosed as one in which the defeasible generalization is improperly treated as an absolute universal generalization of the kind that might be modeled by the universal quantifier in classical deductive logic. It is not subject to exceptions, and an argument based on it as a premise is defeated by one counter-example to the generalization. The error in this kind of case is not easy to evaluate, because we're told very little about the background dialog of how the arguer arrived at the singular conclusion, starting from the generalization expressed in the premise. The leap from such a broad generalization to a singular case to is so lacking in supporting evidence of a kind that would be required to meet the burden of proof that the argument is well classified under the category of fallacy of hasty generalization. However, this fallacy is an error at least partly because it fits the more general category of jumping to a conclusion prematurely on the basis of insufficient evidence. Even worse, it may involve suppression of evidence, a topic we return to in Section 7.

\section{The principle of tutiorism and implicit assumptions}

In both versions 1 and 2 of the Little Dog argument, a key part of the argument is the presumption revealed in the analysis above as the principle of tutiorism. In version 1 , the exception is explicitly stated. It already exists as a premise in the argument, and failure to take it into account is an obvious sort of error. Version 2 is more subtle, and it is not a straightforward matter to judge whether it should properly be said to commit a fallacy of jumping too hastily to a conclusion. In this case, there is an unstated exception to the rule that is not properly taken account of, especially once the implicit presumption of the principle of tutiorism comes into play. This case is more complicated, because implicit premises need to be revealed, and it needs to be shown how they can be used to build a counter-argument that undercuts the original one by postulating an exception to the generalization that is one premise in the original argument. We conclude by offering the comment that it appears dubious whether it would represent a kind of counter-argument that should be classified as meeting the requirements for the fallacy of leaping too hastily to a conclusion. Version 1 of the Little Dog argument shows evidence of all four kinds of error that can occur when an argument leaps too quickly to a conclusion, as represented by Figures 3 through 6.

Here we proposed that the principle of tutiorism could be better modeled as a design principle for argumentation schemes. It works to modify burden of proof in cases of practical deliberation where making 
an error can be dangerous and where negative consequences of accepting the conclusion of an argument are significant and need to be considered. In such a case we may have a choice between accepting two conclusions where one is more plausible than the other. If the more plausible conclusion has significant negative consequences that pose a threat to safety, this consideration may introduce a reason for accepting the less plausible conclusion. However, deciding whether matters of safety should be taken into account at the closing stage of the deliberation where all the arguments on both sides are being weighed up and compared, is a problem that needs to be resolved at the opening stage. Hence, in such a case, the question whether a given argument should be judged to be fallacious depends on procedural considerations.

A different kind of error is involved in the example categorized under the heading of post hoc in Section 1. In this case, a special argumentation scheme is involved (in the version of post hoc we considered), namely that for argument from correlation to cause, and the error is that of overlooking a critical question that should be considered, before leaping to the conclusion that we must eliminate all the laws in order to reduce crime. As in the previous example, the conclusion is such a broad and implausible generalization that the burden of proof required to establish it surely must be set at a high level. The reason that the evidence given in the existing premise is insufficient to establish that conclusion, however, is that it is not easy to argue from correlation to causation, and the additional implicit premises required in order to support such a defeasible argument need also to be supported in order to give the argument any plausibility. Similarly, in the classic foreign spy case, the problem is the overlooking of an implicit premise that requires support in the given case if the argument is to be judged as very plausible.

\section{Suppressing and ignoring evidence}

These considerations bring us to the question of whether there really should be a fallacy of suppressed evidence, of the kind suggested by the example of the Little Dog case, or whether the argument in this case should merely be dismissed as a defeasible argument that is too weak to support its conclusion. What has been shown is that a careful distinction needs to be drawn between two kinds of error. One is committed by a defeasible argument that jumps to a conclusion too quickly by not taking implicit presumptions into account, and the other by a defeasible argument that hides evidence that already exists in a given case. The first kind of error is merely the overlooking of evidence, or not taking it into account in arriving at a conclusion. Version 1 of the Little Dog argument is the key example illustrating this error. In the other kind of case, the fault in the argument is that it moves ahead to a conclusion without taking into account implicit principles that should be seen as presumptions. For example, there are commonly implicit presumptions 
concerning safety, that indicate that a burden of proof should be set in place that the given argument would need to meet. If the little dog is identified as a pit bull, and it is known or accepted that pit bulls are dangerous, suppressing or ignoring this evidence and moving to the conclusion to pet the dog is an even more serious error. If it doesn't meet the burden imposed by tutiorism, the argument should be judged as not acceptable. If there is no evidence whether the little dog is friendly or poses a threat, one kind of error of leaping to the conclusion to pet it is the failure to take into account the possibility of an exception to the rule that dogs are generally friendly. This kind of case clearly involves defeasibility, because even though the general rule holds, there are exceptions to it, and a presumption concerning safety could open the original argument to critical questioning, and then by means of burden of proof, defeat the original argument by undercutting it. Version 2 of the Little Dog argument is an example illustrating an even more serious error of overlooking or suppressing actual evidence.

In either event, we see the failure of suppressing evidence as an error that involves a dialog in which one party has access to evidence, but hides that evidence from the other party. Procedural rules of reasoned dialog should provide the respondent with an opportunity to ask critical questions, and in some types of dialog, each party must present relevant evidence to the other (as in a trial in law). The error here is to deny the respondent this right to have access to evidence. Thus to really get to the bottom of this kind of fallacy, we need to see it not just as an inferential error of drawing the wrong conclusion from a set of premises, but at least partly as a procedural error by one party in a dialog cutting off the possibility of acquiring relevant evidence by the other party.

\section{Modeling defeasible reasoning and fallacies in a dialog model}

The analyses of jumping too quickly to a conclusion presented in this paper modeled openness to various kinds of defeat in sequences of defeasible reasoning as an argument proceeds. The model of rational argument developed in the paper assessed incremental growth of evidence in argumentation as new evidence comes in, new evidence that can undercut or defeat the original argument in some cases. Applying this dynamic method of argument evaluation took us beyond the old inferential model of argument merely being a set of fixed propositions and an inference joining them (a set of premises and a conclusion). Instead, as was shown, in order to judge in various kinds of arguments whether the argument leaps too quickly to a conclusion, we need to look at how the argument evolved dynamically, and how the arguer who put it forward reacts to counter-arguments that may defeat it or support it. A 
dialog model, of the kind much in use in argumentation and $\mathrm{AI}^{12}$, can be proposed as a framework that could help address the problem. It a dialog model, an argument is seen as being used by an arguer to remove doubt expressed by a questioner.

In this kind of case, the fallacy is the failure to make some moves in the dialog which would not further the goals of the dialog. In order to prevent this kind of fallacy from occurring, critical questions need to be asked. In other cases, the problem is to understand how it can be fallacious not to reveal an implicit premise. In the spy example, perhaps the premise in question is one of the essential premises of the argumentation scheme, not a critical question that may be left implicit. However, it could be considered fallacious to omit essential premises from an argumentation scheme.

Studying fallacies in a more realistic way demands examining the strengths and weaknesses of defeasible arguments, and we have shown the job of studying them is a worthy undertaking. Defeasible reasoning is typically used in legal argumentation, or in scientific reasoning at the discovery stage, where an arguer is justified in going ahead tentatively to draw an inference to a conclusion provided he or she is open to defeat in an investigation or dialog should new evidence come in. Such arguments are often necessary, and they are commonly used heuristic devices of both everyday and legal reasoning. But they are inherently dangerous, for not only are they subject to defeat as new evidence comes in, they can even be fallacious, sometimes encouraging jumping to a wrong conclusion. As we have shown, jumping to a wrong conclusion is an error, and indeed can be any one of the four kinds of error. The underlying basic failure in all four cases needs to be at least partly seen as a violation of the procedural norms for a dialog that is underway. Much depends on implicit premises that are revealed as an argument proceeds in such a dialog, on how the original argument is critically questioned, and on how the asking of critical questions is anticipated (or not) by that argument as presented. As we have shown, much also depends on matters of burden of proof that should be set at the opening stage of a dialog.

\section{Conclusions}

Attempts to classify fallacies, by fitting groups of fallacies under more general categories, is still at an early stage. The textbooks exhibit little consistency in this regard. The general project seems to depend on a prior classification of argumentation schemes, and that project as well is still at an early stage. The work in this paper is meant to be a first step in any attempt to classify fallacies.

\footnotetext{
${ }^{12}$ See Gordon (1995), Walton (1995), Prakken and Sartor (1996), and BenchCapon and Prakken (2006).
} 
We conclude that jumping to a conclusion should not be treated as a specific fallacy in its own right, but is better seen as an underlying pattern of erroneous reasoning into which various important fallacies and errors fit. The list of these specific fallacies includes the main four we began by identifying: (1) arguing from premises that are insufficient as evidence to prove the conclusion that is supposed to be proved ( failure to meet the standard of proof appropriate for burden of proof in a dialog), (2) fallacious argument from ignorance, or argument from the absence of reasons to the contrary, as the non-fallacious version of it might better be called, (3) constructing a line of argument that ends in a conclusion other than the one that was supposed to be proved in a dialog (irrelevant conclusion), and (4) using defeasible reasoning that is supposed to be open to defeat if new evidence comes in, but failing to be open to an exception to the defeasible generalization that is the basis of the inference. This fourth form of error has been traditionally classified under the heading of the fallacy of hasty generalization, or sometimes even more appropriately we think, the fallacy called secundum quid, referring to a failure to be open to exceptions to a general rule. Another variant on this fourth fallacy is the fallacy of being closed-minded by not only ignoring an exception to a rule but also by actively refusing to countenance the exception, or even its possibility. All these fallacies, and perhaps others as well fit under the more general category of jumping to a conclusion.

We added to this list the faults of overlooking and suppressing evidence. Throughout the paper we saw that there are more simple errors of overlooking an exception and more serious fallacies of failing to admit, or even suppressing an exception. This duality can also be observed in the terminological ambivalence between ignoring evidence and suppressing evidence discussed in Section 7. We think that it is an error to treat ignoring and suppressing evidence as on a par, even though they are closely related failures of reasoned argumentation. Suppression of evidence is a more active effort in a dialog that would seem to almost always pernicious and culpable, whereas ignoring evidence may be neither, even though it can often be a problem in rational thinking. Whether suppression of evidence and ignoring evidence are fallacious in specific cases where they occur, on our view, depends on how they are used in context of dialog.

\section{References}

Aristotle (MCMLX). Topics. Loeb Classical Library. Trans. by E.S. Forster, Cambridge, MA: Harvard University Press.

Atkinson, K. and Bench-Capon, T.J.M. (2007). Argumentation and Standards of Proof. In R. Winkels (Ed.), Proceedings of the Eleventh International Conference on Artificial Intelligence and Law, pp. 107-116. New York: ACM Press. Available at: 


\section{http://www.csc.liv.ac.uk/ katie/icail07.pdf}

Bench-Capon, T.J.M. and Prakken, H. (2006). Argumentation. In A.R.

Lodder \& A. Oskamp (Eds.), Information Technology \& Lawyers: Advanced Technology in the Legal Domain, from Challenges to Daily Routine, pp. 61-80. Berlin: Springer Verlag.

Carey, S.S. (2000). The Uses and Abuses of Argument. Mountain View, CA: Mayfield Publishing Company.

Cicero, M.T. (1933). Academica. Loeb Classical Library. Cambridge, Mass: Harvard University Press.

Freeman, K. and Farley, A. (1996). A Model of Argumentation and Its Application to Legal Reasoning. Artificial Intelligence and Law, 4, 163-197.

Gordon, T.F. (1995). The Pleadings Game: An Artificial Intelligence Model of Procedural Justice. Dordrecht: Kluwer.

Gordon, T.F. Prakken, H. and Walton, D. (2007). The Carneades Model of Argument and Burden of Proof. Artificial Intelligence, 171, 875896.

Gordon, T.F. and Walton, D. (2009). Proof Standards and Burden of Proof. In I. Rahwan \& G. Simari (Eds.). Argumentation in Artificial Intelligence. Berlin: Springer.

Hamblin, C.L. (1970). Fallacies. London: Methuen.

Horty, J. (2001). Nonmonotonic Logic. In L. Goble (Ed.), The Blackwell Guide to Philosophical Logic, pp. 336-361. Oxford: Blackwell.

Hurley, P. (2003). A Concise Introduction to Logic, $8^{\text {th }}$ ed. Belmont, CA: Wadsworth.

Johnson, R.H. and Blair, J.A. (1977). Logical Self-Defense. $1^{\text {st }}$ ed. Toronto: McGraw-Hill Ryerson.

Jonsen, A.R. Toulmin, S. (1988). The Abuse of Casuistry: A History of Moral Reasoning. Berkeley: University of California Press.

Kahane, H. (1971). Logic and Contemporary Rhetoric. Belmont, CA: Wadsworth.

Pinto, R.C. (1995). Post Hoc Ergo Propter Hoc. In H.V. Hansen \& R.C. Pinto (Eds.), Fallacies: Classical and Contemporary Readings, pp. 302-311. University Park, PA: The Pennsylvania State University Press.

Pollock, J. (1995). Cognitive Carpentry. Cambridge, MA: MIT Press.

Prakken, H. and Sartor, G. (1996). A Dialectical Model of Assessing Conflicting Arguments in Legal Reasoning. Artificial Intelligence and Law, 4, 331-368.

Prakken, H. and Sartor, G. (1997). Argument-based Extended Logic Programming with Defeasible Priorities. Journal of Applied Nonclassical Logics, 7, 25-75.

Reed, C. and Rowe, G. (2004). Araucaria: Software for Argument Analysis, Diagramming and Representation. International Journal of AI Tools, 13, 961-980.

Reed, C. and Rowe, G. (2005). Araucaria, Version 3. Available free at http://www.computing.dundee.ac.uk/staff/creed/araucaria/. 
Reiter, R. (1980). A Logic for Default Reasoning. Artificial Intelligence, $13,81-132$.

Walton, D. (1989). Informal Logic. Cambridge: Cambridge University Press.

Walton, D. (1995). A Pragmatic Theory of Fallacy. Tuscaloosa, AB: University of Alabama Press.

Walton, D. (1996). Arguments from Ignorance. University Park, PA: The Pennsylvania State University Press.

Walton, D. (1999). Rethinking the Fallacy of Hasty Generalization. Argumentation, 13, 161-182.

Walton, D. (2004). Relevance in Argumentation. Mahwah, NJ: Lawrence Erlbaum.

Walton, D. (2006). Fundamentals of Critical Argumentation. Cambridge: Cambridge University Press.

Walton, D. and Reed, C. (2002). Argumentation Schemes and Defeasible Inferences. In G. Carenini, F. Grasso \& C. Reed (Eds.), Working Notes of the ECAI 2002 Workshop on Computational Models of Natural Argument, pp. 45-55. Available at:

http://www.csc.liv.ac.uk/ floriana/CMNA/WaltonReed.pdf 